\title{
Lie nilpotency indices of symmetric elements under oriented involutions in group algebras
}

\section{John H. Castillo}

Departamento de Matemáticas y Estadística, Universidad de Nariño

E-mail address: jhcastillo@udenar.edu.co--jhcastillo@gmail.com

\begin{abstract}
Let $G$ be a group and let $F$ be a field of characteristic different from 2. Denote by $(F G)^{+}$the set of symmetric elements and by $\mathcal{U}^{+}(F G)$ the set of symmetric units, under an oriented classical involution of the group algebra $F G$. We give some lower and upper bounds on the Lie nilpotency index of $(F G)^{+}$and the nilpotency class of $\mathcal{U}^{+}(F G)$.
\end{abstract}

\section{Introduction}

Let $F G$ denote the group algebra of a group $G$ over a field $F$ with $\operatorname{char}(F)=$ $p \neq 2$. A homomorphism $\sigma: G \rightarrow\{ \pm 1\}$ is called an orientation of the group $G$. Working in the context of $K$-theory, Novikov [11], introduced an oriented involution $*$ of $F G$, given by

$$
\left(\sum_{g \in G} \alpha_{g} g\right)^{*}=\sum_{g \in G} \alpha_{g} \sigma(g) g^{-1} .
$$

When $\sigma$ is trivial this involution coincides with the so called classical involution of $F G$.

We denote $(F G)^{+}=\left\{\alpha \in F G: \alpha^{*}=\alpha\right\}$ and $(F G)^{-}=\left\{\alpha \in F G: \alpha^{*}=\right.$ $-\alpha\}$ the set of symmetric and skew-symmetric elements of $F G$ under *, respectively. We denote by $N$ the kernel of $\sigma$. It is obvious that the involution $*$ coincides on the group algebra $F N$ with the classical involution.

2010 Mathematics Subject Classification. 16W10, 16U80, $16 \mathrm{U} 60$.

Key words: Involution, symmetric elements, Lie nilpotent, strongly Lie nilpotent, Lie nilpotency index, nilpotency class. 
It is easy to see that, as an $F$-module, $(F G)^{+}$is generated by the set

$$
\mathcal{S}=\left\{g+g^{-1}: g \in N\right\} \cup\left\{g-g^{-1}: g \in G \backslash N, g^{2} \neq 1\right\}
$$

and $(F G)^{-}$is generated by

$$
\mathcal{L}=\left\{g+g^{-1}: g \in G \backslash N\right\} \cup\left\{g-g^{-1}: g \in N, g^{2} \neq 1\right\} .
$$

Given $g_{1}, g_{2} \in G$, we define the commutator $\left(g_{1}, g_{2}\right)=g_{1}^{-1} g_{2}^{-1} g_{1} g_{2}$ and recursively,

$\left(g_{1}, \ldots, g_{n}\right)=\left(\left(g_{1}, \ldots, g_{n-1}\right), g_{n}\right)$ for $n$ elements $g_{1}, \ldots, g_{n}$ of $G$. By the commutator $(X, Y)$ of the subsets $X$ and $Y$ of $G$ we mean the subgroup of $G$ generated by all commutators $(x, y)$ with $x \in X, y \in Y$. In this way, we can define the lower central series of a nonempty subset $H$ of $G$ by: $\gamma_{1}(H)=H$ and $\gamma_{n+1}(H)=\left(\gamma_{n}(H), H\right)$, for $n \geq 1$. We say that $H$ is nilpotent if $\gamma_{n}(H)=1$, for some $n$. For a nilpotent subset $H \subseteq G$ the number $\operatorname{cl}(H)=\min \left\{n \in \mathbb{N}_{0}: \gamma_{n+1}(H)=1\right\}$ is called the nilpotency class of $H$. It can be proved that $H$ is a nilpotent set if and only if $H$ satisfies the group identity $\left(g_{1}, \ldots, g_{n}\right)=1$ for some $n \geq 2$.

In an associative ring $R$, the Lie bracket on two elements $x, y \in R$ is defined by $[x, y]=x y-y x$. This definition is extended recursively via $\left[x_{1}, \ldots, x_{n+1}\right]=\left[\left[x_{1}, \ldots, x_{n}\right], x_{n+1}\right]$. For $X, Y \subseteq R$ by $[X, Y]$ we denote the additive subgroup generated by all Lie commutators $[x, y]$ with $x \in X, y \in$ $Y$. The lower Lie central series of a nonempty subset $S$ of $R$ is defined inductively by setting $\gamma^{1}(S)=S$ and $\gamma^{n+1}(S)=\left[\gamma^{n}(S), S\right]$. We say that the subset $S$ is Lie nilpotent if there exists a natural number $n$, such that $\gamma^{n}(S)=0$. The smallest natural number with the last property, denoted by $t(S)$, is called the Lie nilpotency index of $S$. It is possible to show that $S$ is Lie nilpotent if and only if $S$ satisfies the polynomial identity $\left[x_{1}, \ldots, x_{n}\right]=0$ for some $n \geq 2$.

Given a nonempty subset $S$ of $R$, we let $S^{(1)}=R$, and then for each $i \geq 2$, let $S^{(i)}$ be the (associative) ideal of $R$ generated by all elements of the form $[a, b]$, with $a \in S^{(i-1)}, b \in S$. We say that $S$ is strongly Lie nilpotent if $S^{(i)}=0$ for some $i$. The minimal $n$ for which $S^{(n)}=0$ is called the upper Lie nilpotency index and denoted by $\mathrm{t}^{\mathrm{L}}(S)$. Clearly, strong Lie nilpotence implies Lie nilpotence and $\mathrm{t}(S) \leq \mathrm{t}^{\mathrm{L}}(S)$. Denote by $\mathcal{U}(S)$ the set of units in the subset $S$ of $R$ and suppose that it is nonempty. By the equality $(x, y)=1+x^{-1} y^{-1}[x, y]$, it is easy to see that $\gamma_{n}(\mathcal{U}(S)) \subseteq 1+S^{(n)}$ for all $n \geq 2$. In consequence, the set of units of a strongly Lie nilpotent subset $S$ is nilpotent, and

$$
\operatorname{cl}(\mathcal{U}(S))<\mathrm{t}^{\mathrm{L}}(S) .
$$

In 1973, Passi, Passman and Sehgal [12] showed that the group algebra $F G$ is Lie nilpotent if and only if $G$ is nilpotent and $G^{\prime}$ is a finite $p$-group, 
where $p$ is the characteristic of $F$. Actually, see [14, a group algebra is Lie nilpotent if and only if it is strongly Lie nilpotent. Next, S.K. Sehgal characterized group algebras which are Lie $n$-Engel, for some $n$.

In 1993, Giambruno and Sehgal [6] began the study of Lie nilpotence of symmetric and skew-symmetric elements under the classical involution. They proved that given a group $G$ without elements of order 2 and a field $F$ with $\operatorname{char}(F) \neq 2$, if either $(F G)^{+}$or $(F G)^{-}$is Lie nilpotent, then $F G$ is Lie nilpotent. This work was completed by G.T. Lee [8], for groups in general. More specifically, he proved that the Lie nilpotence of the symmetric elements under the classical involution is equivalent to the Lie nilpotence of $F G$ when the group $G$ does not contain a copy of $Q_{8}$, the quaternion group of order 8 and he also characterized the group algebras such that the set of symmetric elements is Lie nilpotent when $G$ contains a copy of $Q_{8}$.

Recently, Castillo and Polcino Milies, see [5], studied Lie properties of the symmetric elements under an oriented classical involution. They extended some previous results from [6], 8] and [9]. In particular, they gave some groups algebras such that the Lie nilpotence of the symmetric set implies the same property in the whole group algebra. Also, they obtained a complete characterization of the group algebras $F G$, such that $Q_{8} \subseteq G$ and $(F G)^{+}$is Lie nilpotent.

Lately, Z. Balogh and T. Juhász in [2] and [3] studied the Lie nilpotency index of $(F G)^{+}$and the nilpotency class of the $\mathcal{U}^{+}(F G)$ under the classical involution in group algebras. They gave a necessary condition to the numbers $\mathrm{t}\left((F G)^{+}\right)$and $\operatorname{cl}\left(\mathcal{U}^{+}(F G)\right)$ be maximal, as possible, in a nilpotent group algebra. Also, they studied this two numbers to group algebras such that $(F G)^{+}$is Lie nilpotent but $F G$ is not.

In this article we study the Lie nilpotency index of $(F G)^{+}$and the nilpotency class of $\mathcal{U}^{+}(F G)$ under an oriented classical involution. In the next section we give some preliminary results. In the third section we study the numbers $\mathrm{t}\left((F G)^{+}\right)$and $\operatorname{cl}\left(\mathcal{U}^{+}(F G)\right)$ in Lie nilpotent group algebras. In the fourth section we study the case when $Q_{8} \subseteq G$ and $(F G)^{+}$is Lie nilpotent. Throughout this paper $F$ will always denote a field of characteristic not 2, $G$ a group and $\sigma$ a nontrivial orientation of $G$. In a number of places, all over this paper, we use arguments from [2], 3] and [10]. Some of them are reproduced here for the sake of completeness.

\section{Preliminaries}

We recall the following result from [10. 
Lemma 2.1. Let $R$ be a ring and $S$ a subset of $R$. Suppose, for some $i \geq 1$, that $S^{(i)} \subseteq z R$, where $z$ is central in $R$. Then for all $j>0$, we have $S^{(i+j)} \subseteq z S^{(j)}$. In particular, for any positive integer $m, S^{(m i)} \subseteq z^{m} R$.

Proof. The proof is by induction on $j$. If $j=1$, then $S^{(i+1)} \subseteq S^{(i)}$, there is nothing to do. Assume that $S^{(i+j)} \subseteq z S^{(j)}$. Take $a \in S^{(i+\bar{j})}, b \in S$. So $a=z a_{1}$, for some $a_{1} \in S^{(j)}$. Thus, $[a, b]=\left[z a_{1}, b\right]=z\left[a_{1}, b\right] \in z S^{(i+j)}$, as we want to prove.

To get the second part, notice that

$$
S^{(2 i)}=S^{(i+i)} \subseteq z S^{(i)} \subseteq z^{2} R .
$$

Suppose that $S^{((m-1) i)} \subseteq z^{m-1} R$. So $S^{(m i)}=S^{((m-1) i+i)} \subseteq z S^{((m-1) i)} \subseteq$ $z^{m} R$.

Throughout this article we denote by $Q_{8}=\left\langle x, y: x^{4}=1, x^{2}=y^{2}, x^{y}=x^{-1}\right\rangle$ the quaternion group of order 8. Castillo and Polcino Milies [5] characterized the group algebras of groups containing $Q_{8}$ and with a nontrivial orientation, such that $(F G)^{+}$is Lie nilpotent. Here we prove that the conditions obtained by them are also satisfied when $(F G)^{+}$is strongly Lie nilpotent.

Theorem 2.1. Let $F$ be a field of characteristic $p \neq 2, G$ a group with a nontrivial orientation $\sigma$ and $x, y$ elements of $G$ such that $\langle x, y\rangle \simeq Q_{8}$. Then $(F G)^{+}$is strongly Lie nilpotent if and only if either

(i) $\operatorname{char}(F)=0, N \simeq Q_{8} \times E$ and $G \simeq\left\langle Q_{8}, g\right\rangle \times E$, where $E^{2}=1$ and $g \in G \backslash N$ is such that $(g, x)=(g, y)=1$ and $g^{2}=x^{2}$; or,

(ii) $\operatorname{char}(F)=p>2, N \simeq Q_{8} \times E \times P$, where $E^{2}=1, P$ is a finite p-group and there exists $g \in G \backslash N$ such that $G \simeq\left\langle Q_{8}, g\right\rangle \times E \times P$, $(g, x)=(g, y)=1$ and $g^{2}=x^{2}$.

Proof. If $(F G)^{+}$is strongly Lie nilpotent, then $(F G)^{+}$is Lie nilpotent and from [5, Theorem 4.2] we get (i) and (ii).

Conversely, assume that $|P|=p^{n}$. We claim that, $\left((F G)^{+}\right)^{\left(2 p^{n}\right)}=0$. The proof will be by induction on $n$. If $n=0$, then $G \simeq\left\langle Q_{8}, g\right\rangle \times E$ and thus, from [5, Lemma 4.3], $(F G)^{+}$is commutative. Assume that $|P|=p^{n}>1$. Take $z \in \zeta(P)$ with $o(z)=p$, applying our inductive hypothesis on $\bar{G}=$ $G /\langle z\rangle$. Then, $\left((F \bar{G})^{+}\right)^{\left(2 p^{n-1}\right)}=0$. Thus

$$
\left((F G)^{+}\right)^{\left(2 p^{n-1}\right)} \subseteq \Delta(G,\langle z\rangle)=(z-1) F G .
$$

By Lema 2.1.

as we claimed.

$$
\left((F G)^{+}\right)^{\left(2 p^{n}\right)} \subseteq(z-1)^{p} F G=0,
$$

São Paulo J.Math.Sci. 7, 1 (2013), 6981 
From the equality, $(x, y)=1+x^{-1} y^{-1}[x, y]$ we know that $\gamma_{n}\left(\mathcal{U}^{+}(F G)\right) \subseteq$ $1+\left((F G)^{+}\right)^{(n)}$ and thus we get the following.

Corollary 2.1. Let $F$ be a field of characteristic different from 2. Assume that $Q_{8} \subseteq G$ and $(F G)^{+}$is Lie nilpotent. Then, $\mathcal{U}^{+}(F G)$ is nilpotent.

We need the following easy observation.

Lemma 2.2. Let $G$ be a group, $H$ any subgroup and $A$ a normal subgroup such that $A \subseteq N$. If $(F G)^{+}$is Lie nilpotent, then so are $(F H)^{+}$and $(F(G / A))^{+}$. Furthermore, $\mathrm{t}\left((F H)^{+}\right) \leq \mathrm{t}\left((F G)^{+}\right)$and $\mathrm{t}\left((F(G / A))^{+}\right) \leq$ $\mathrm{t}\left((F G)^{+}\right)$.

Proof. Note that $(F H)^{+}$is a subset of $(F G)^{+}$, and thus it has the required properties.

Since $A$ is a normal subgroup contained in the kernel of the orientation $\sigma$, we can define in $F(G / A)$ an induced oriented classical involution from $*$ in $F G$ as follows:

$$
\left(\sum_{\bar{g} \in G / A} \alpha_{g} \bar{g}\right)^{\star}=\sum_{\bar{g} \in G / A} \alpha_{g} \sigma(g) \bar{g}^{-1} .
$$

Now, simply observe that the symmetric elements in $F(G / A)$, under $\star$, are linear combinations of terms of the form $g A+\sigma(g) g^{-1} A$, with $g \in G$. That is, every element of $(F(G / A))^{+}$is the homomorphic image of an element of $(F G)^{+}$under the natural map $\varepsilon_{A}: F G \rightarrow F(G / A)$, defined by $\varepsilon_{A}\left(\sum_{g \in G} \alpha_{g} g\right)=\sum_{g \in G} \alpha_{g} \bar{g}$.

So assume that $(F G)^{+}$is Lie nilpotent, therefore there exists $n=\mathrm{t}\left((F G)^{+}\right)$ such that $\left[\alpha_{1}, \ldots, \alpha_{n}\right]=0$ for all $\alpha_{i} \in(F G)^{+}$. Let $\beta_{1}, \ldots, \beta_{n} \in(F(G / A))^{+}$. Thus

$$
\begin{aligned}
{\left[\beta_{1}, \ldots, \beta_{n}\right] } & =\left[\varepsilon_{A}\left(\alpha_{1}\right), \ldots, \varepsilon_{A}\left(\alpha_{n}\right)\right] \\
& =\varepsilon_{A}\left(\left[\alpha_{1}, \ldots, \alpha_{n}\right]\right)=\varepsilon_{A}(0)=0 .
\end{aligned}
$$

Consequently, $\mathrm{t}\left((F(G / A))^{+}\right) \leq \mathrm{t}\left((F G)^{+}\right)$.

\section{Lie nilpotent group algebras}

In this section we assume that $F G$ is Lie nilpotent. By [15], $\mathrm{t}^{\mathrm{L}}(F G) \leq$ $\left|G^{\prime}\right|+1$ and by [4] the equality holds if and only if $G^{\prime}$ is cyclic, or $G^{\prime}$ is a noncentral elementary abelian group of order 4 . 
Note that a group $G$ of odd finite order has trivial orientation. Indeed, let $a$ be an element of $G$. So $1=\sigma\left(a^{|G|}\right)=\sigma(a)^{|G|}$ and as $|G|$ is odd we get that $\sigma(a)=1$. For the last reason when $G$ is a group of odd finite order, the involution $*$ is the classical involution. In this way, we can use the following result, that is a combination from [2, Lemma 2] and [3, Lemma $2]$.

Lemma 3.1. Let $G$ be a finite p-group with a cyclic derived subgroup. Then $\mathrm{t}\left((F G)^{+}\right) \geq\left|G^{\prime}\right|+1$ and $\operatorname{cl}\left(\mathcal{U}^{+}(F G)\right) \geq\left|G^{\prime}\right|$.

We recall that a group $G$ is called $p$-abelian if $G^{\prime}$, the commutator subgroup of $G$, is a finite $p$-group and 0 -abelian means abelian.

Theorem 3.1. Let $F G$ be a Lie nilpotent group algebra of odd characteristic and nontrivial orientation. Then, $\mathrm{t}\left((F G)^{+}\right)=\left|G^{\prime}\right|+1$ if and only if $G^{\prime}$ is cyclic. Moreover, assuming that $G$ is a torsion group, $\operatorname{cl}\left(\mathcal{U}^{+}(F G)\right)=\left|G^{\prime}\right|$ if and only if $G^{\prime}$ is cyclic.

Proof. Assume that $\mathrm{t}\left((F G)^{+}\right)=\left|G^{\prime}\right|+1$. As $G^{\prime}$ is a finite $p$-group, if $G^{\prime}$ is not cyclic, from [4], we know that $t\left((F G)^{+}\right) \leq \mathrm{t}^{\mathrm{L}}(F G)<\left|G^{\prime}\right|+1$ and we get a contradiction. Thus, $G^{\prime}$ is cyclic.

Conversely, suppose that $G^{\prime}$ is cyclic. By the hypotheses, $G$ is a nilpotent $p$-abelian group and from [1, Lemma 1] there exists a finite $p$-group $P$ which is isomorphic to a subgroup of factor group of $G$ and $P^{\prime} \simeq G^{\prime}$. Actually, from the proof of [1, Lemma 1], we know that $P \simeq H / A$, where $A$ is a maximal torsion-free central subgroup of $G$.

Assume that there exists $g \in A$ such that $\sigma(g)=-1$. In this way, as $G=N \cup g N$, we get $G^{\prime}=N^{\prime}$. Using in FP the classical involution, by lemmas 3.1 and 2.2 , we obtain that

$$
\left|G^{\prime}\right|+1=\left|N^{\prime}\right|+1=\left|P^{\prime}\right|+1 \leq \mathrm{t}\left((F P)^{+}\right) \leq \mathrm{t}\left((F N)^{+}\right) \leq \mathrm{t}\left((F G)^{+}\right) .
$$

In the other hand, suppose that $A \subseteq N$. Then we can define an induced oriented classical involution in $P \simeq H / A$, from that one in $F G$. Consequently,

$$
\left|G^{\prime}\right|+1=\left|P^{\prime}\right|+1 \leq \mathrm{t}\left((F P)^{+}\right) \leq \mathrm{t}\left((F G)^{+}\right) .
$$

The proof of the second part is similar.

\section{Groups that contain a copy of $Q_{8}$}

We assume that $Q_{8} \subseteq G$ and $(F G)^{+}$is Lie nilpotent. This means that the group algebra $F G$ is not Lie nilpotent. Recently, this kind of group algebras was characterized by Castillo and Polcino Milies [5]. This characterization is the same as in Theorem 2.1, so during this section we assume that $G$ is 
as in that result. In this section, we will study the Lie nilpotency index of the symmetric elements under oriented classical involutions.

It is easy to show that

$$
g^{m}-1 \equiv m(g-1) \quad\left(\bmod \Delta(G)^{2}\right) .
$$

for every $g \in G$ and any integer $m$.

We begin with the following result.

Lemma 4.1. Consider FG with an oriented classical involution. Then

$$
\left((F G)^{+}\right)^{(n)} \subseteq F G \Delta(P)^{n}
$$

for all $n \geq 2$

Proof. Recall that the symmetric elements are spanned as an $F$-module by the set

$$
\mathcal{S}=\left\{z+z^{-1}: z \in N\right\} \cup\left\{z-z^{-1}: z \in G \backslash N\right\} .
$$

If $z \in N$, then $z=a h$ with $a \in Q_{8} \times E$ and $h \in P$. Note that if $a^{2} h=1$, then $h=1$ and $a^{2}=1$. Thus, $a \in \zeta\left(Q_{8} \times E\right)$. Assuming $a^{2} h \neq 1$, follows that $z+z^{-1}=a h+a^{-1} h^{-1}=a h+a^{3} h^{-1}=a\left(h+a^{2} h^{-1}\right)$.

Also, if $z \in G \backslash N$; we can write $z=$ gah with $a \in Q_{8} \times E$ and $h \in P$. If $a^{2} h=1$, then $a^{2}=h=1$. Again, $a \in \zeta\left(Q_{8} \times E\right)$ and thus $z-z^{-1}=$ $g a h-g^{-1} a^{-1} h^{-1}=g a-g^{-1} a=g a\left(1-g^{2}\right) \in \zeta\left(Q_{8} \times E\right)$. Now we suppose that $a^{2} h \neq 1$ and we get the following cases:

(1) If $a^{2}=1$ and $h \neq 1$, then $z-z^{-1}=g a h-g^{-1} a^{-1} h^{-1}=a g(h-$ $\left.g^{2} h^{-1}\right)$.

(2) If $a^{2} \neq 1$ and $h=1$, then $z-z^{-1}=g a h-g^{-1} a^{-1} h^{-1}=g a-g^{3} a^{3}=$ $g a-g a=0$.

(3) If $a^{2} \neq 1$ and $h \neq 1$, then $z-z^{-1}=g a h-g^{-1} a^{-1} h^{-1}=a g h-$ $a^{3} g^{3} h^{-1}=a g\left(h-h^{-1}\right)$, because $a^{3} g^{3}=a g$.

From the above considerations, we obtain that

$$
\mathcal{S}=\mathcal{A} \cup \mathcal{B} \cup \mathcal{C} \cup \zeta\left(Q_{8} \times E\right),
$$

where

$$
\begin{aligned}
& \mathcal{A}=\left\{a\left(h+a^{2} h^{-1}\right): a \in Q_{8} \times E, h \in P \text { and } a^{2} h \neq 1\right\}, \\
& \mathcal{B}=\left\{a g\left(h-g^{2} h^{-1}\right): a \in Q_{8} \times E, h \in P \text { and }\left(a^{2}=1 \text { and } h \neq 1\right)\right\}, \\
& \mathcal{C}=\left\{a g\left(h-h^{-1}\right): a \in Q_{8} \times E, h \in P \text { and }\left(a^{2} \neq 1 \text { and } h \neq 1\right)\right\} .
\end{aligned}
$$

Given $a \in Q_{8} \times E$, such that $a^{2} \neq 1$ we know that $1+a^{2}$ is symmetric and $a^{2} \in \zeta\left(Q_{8} \times E\right)$. In this way,

$$
a\left(h+a^{2} h^{-1}\right)+1+a^{2}=a(h-1)+a^{3}\left(h^{-1}-1\right)+1+a+a^{2}+a^{3},
$$


where $1+a+a^{2}+a^{3}$ is a central element in $F G$ and $a(h-1)+a^{3}\left(h^{-1}-1\right) \in$ $F G \Delta(P)$. It is clear that, $a g\left(h-h^{-1}\right) \in F G \Delta(P)$. Furthermore, if $a^{2}=1$ and $h \neq 1$, then $a g\left(h-g^{2} h^{-1}\right)=a g(h-1)-a g^{3}\left(h^{-1}-1\right)+a\left(g-g^{-1}\right) \in$ $F G \Delta(P)+\zeta(F G)$.

So

$$
\widetilde{\mathcal{S}}=\mathcal{A}^{\prime} \cup \mathcal{B} \cup \mathcal{C} \cup \zeta\left(Q_{8} \times E\right),
$$

also spans $(F G)^{+}$as an $F$-module, where

$$
\mathcal{A}^{\prime}=\left\{a\left(h+a^{2} h^{-1}\right)+1+a^{2}: a \in Q_{8} \times E, h \in P \text { and } a^{2} h \neq 1\right\}
$$

and $\mathcal{B}, \mathcal{C}$ are as above.

In consequence,

$$
(F G)^{+} \subseteq F G \Delta(P)+\zeta(F G) .
$$

The proof follows by induction on $n$. Indeed, if $n=2$

$$
\left[(F G)^{+},(F G)^{+}\right] \subseteq[F G \Delta(P), F G \Delta(P)] \subseteq F G \Delta(P)^{2} .
$$

Suppose that the lemma is true for some $n \geq 2$. Take $\alpha \in\left((F G)^{+}\right)^{(n)}$ and $\beta \in(F G)^{+}$. So

$$
[\alpha, \beta] \in\left[F G \Delta(P)^{n}, F G \Delta(P)\right] \subseteq F G \Delta(P)^{n+1} .
$$

and we get that $\left((F G)^{+}\right)^{(n+1)} \subseteq F G \Delta(P)^{n+1}$ as required.

Denote by $c$ the central element of $Q_{8} \times E$, such that $\left(Q_{8} \times E\right)^{2}=\langle c\rangle$. Given $n \geq 2$, we denote with $M_{n}$ the $F$-subspace of the vector space $F G$ generates by the set

$\left\{\left(h_{1}-h_{1}^{-1}\right) \cdots\left(h_{n}-h_{n}^{-1}\right)(1-c) a: h_{1}, \ldots, h_{n} \in P, a \in\left(Q_{8} \times E\right) \backslash \zeta\left(Q_{8} \times E\right)\right\}$.

To simplify, we write $f_{1, \ldots, n}$ instead of $\left(h_{1}-h_{1}^{-1}\right) \cdots\left(h_{n}-h_{n}^{-1}\right)$.

Let $S_{n}$ be the symmetric group of degree $n$ and $F S_{n}$ its group algebra over the field $F$. It is possible to define a group action of $S_{n}$ on $M_{n}$ via: for a $\sigma \in S_{n}$ and a generator element $f_{1, \ldots, n}(1-c) a$ of $M_{n}$ let

$$
\sigma \cdot f_{1, \ldots, n}(1-c) a=f_{\sigma(1), \ldots, \sigma(n)}(1-c) a .
$$

Naturally, this group action on a generator set of $M_{n}$ can be extended linearly to the whole $M_{n}$. We extend this group action to a group algebra action: for $x=\sum_{\sigma \in S_{n}} \alpha_{\sigma} \sigma \in F S_{n}$ and $z \in M_{n}$, let

$$
x \cdot z=\sum_{\sigma \in S_{n}} \alpha_{\sigma}(\sigma \cdot z) .
$$

São Paulo J.Math.Sci. 7, 1 (2013), 6981 
For $n \geq 2$ we define the elements $x_{2, n}, x_{3, n}, \ldots, x_{n, n}$ of $F S_{n}$ recursively as:

$$
\begin{aligned}
& x_{2, n}=1+(2,1), \\
& x_{i, n}=x_{i-1, n}+x_{i-1, n}(i, i-1, \ldots, 1) ; \text { for } 3 \leq i \leq n .
\end{aligned}
$$

Since $(F N)^{+} \subseteq(F G)^{+}$, from Lemma 4 and Lemma 5 in [3], we get the following results.

Lemma 4.2. $x_{n, n} M_{n} \in \gamma^{n}\left((F G)^{+}\right)(1-c)$ for all $n \geq 2$.

Lemma 4.3. If $|P|=p^{k}$, then $\widehat{P}(1-c) a \in \gamma^{k(p-1)}\left((F G)^{+}\right)$for some $a \in Q_{8} \times E$

We recall that the augmentation ideal $\Delta(P)$ of a finite $p$-group $P$ is a nilpotent ideal, see [13, Theorem 6.3.1], we will denote by $t_{n i l}(P)$ its nilpotency index. Also, we remind that a finite $p$-group $P$, is called powerful if $P^{\prime} \subseteq P^{p}$. Let $P$ be a powerful group. We denote with $D_{i}=D_{i}(F P)$ the $i$-th dimensional subgroup. By Theorem 5.5 in [7], $D_{1}=P$ and for $n>1$,

$$
D_{n}=\left\langle\left(D_{n-1}, P\right),\left(D_{\left\lceil\frac{n}{p}\right\rceil}\right)^{p}\right\rangle .
$$

It can be showed that, $\left(P^{p^{i}}\right)^{p^{j}}=P^{p^{i+j}}$ and $\left(P^{p^{i}}, P\right) \subseteq P^{p^{i+1}}$ for every pair $i, j$. So, if $p^{i-1}<n \leq p^{i}$ then $D_{n}=P^{p^{i}}$.

Lemma 4.4. Let $P$ be a powerful group and $h_{i}-1 \in \Delta(P)^{k_{i}}$ and $h_{j}-1 \in$ $\Delta(P)^{k_{j}}$, where $k_{i}$ and $k_{j}$ are positive integers. Then

$$
\left(h_{i}-1\right)\left(h_{j}-1\right) \equiv\left(h_{j}-1\right)\left(h_{i}-1\right) \quad\left(\bmod \Delta(P)^{k_{i}+k_{j}+1}\right) .
$$

Proof. First, we prove that $\left(D_{i}, D_{j}\right) \subseteq D_{i+j+1}$, for every $i, j$. Take $h_{i} \in D_{i}$ and $h_{j} \in D_{j}$. We get the following equation

$$
\left(h_{i}, h_{j}\right)-1=h_{i}^{-1} h_{j}^{-1}\left(\left(h_{i}-1\right)\left(h_{j}-1\right)-\left(h_{j}-1\right)\left(h_{i}-1\right)\right) .
$$

If either $i$ or $j$, say $i$, is not a power of $p$, then $h_{i} \in D_{i}=D_{i+1}$, so by (7), $\left(h_{i}, h_{j}\right)-1 \in \Delta(P)^{i+j+1}$; thus $\left(h_{i}, h_{j}\right) \in D_{i+j+1}$. If both $i$ and $j$ are powers of $p$, then $i+j$ cannot be a power of $p$ and consequently $D_{i+j}=D_{i+j+1}$. By (7) follows $\left(h_{i}, h_{j}\right) \in D_{i+j+1}$; therefore our claim is proved.

Let $h_{i}-1 \in \Delta(P)^{k_{i}}$ and $h_{j}-1 \in \Delta(P)^{k_{j}}$ for some positive integers $k_{i}, k_{j}$. Then

$$
\left(h_{i}-1\right)\left(h_{j}-1\right)=\left(h_{j}-1\right)\left(h_{i}-1\right)+h_{j} h_{i}\left(\left(h_{i}, h_{j}\right)-1\right),
$$

and as $\left(h_{i}, h_{j}\right) \in D_{k_{i}+k_{j}+1}$, the result follows.

Now we can prove our main result in this section. 
Theorem 4.1. Let $F$ be a field of characteristic $p>2$. Consider the group algebra $F G$ with an oriented classical involution. Assume that $Q_{8} \subseteq G$, $(F G)^{+}$is Lie nilpotent and the Sylow p-group $P$ of $G$ is of order $p^{m}$, with $m \geq 1$. Then

(i) $1+m(p-1) \leq \mathrm{t}\left((F G)^{+}\right) \leq \mathrm{t}^{\mathrm{L}}\left((F G)^{+}\right) \leq \mathrm{t}_{\text {nil }}(P)$ and $\operatorname{cl}\left(\mathcal{U}^{+}(F G)\right) \leq$ $\mathrm{t}_{\text {nil }}(P)-1$.

(ii) If $\mathrm{t}\left((F G)^{+}\right)=\mathrm{t}_{\text {nil }}(P)$, then $\operatorname{cl}\left(\mathcal{U}^{+}(F G)\right)+1=\mathrm{t}\left((F G)^{+}\right)$.

(iii) If $P$ is powerful, then $\mathrm{t}\left((F G)^{+}\right)=\mathrm{t}_{\text {nil }}(P)$.

(iv) If $P$ is abelian, then, for all $k \geq 2$, the $F$-space $\gamma^{k}\left((F G)^{+}\right)$is generated by the set

$$
\begin{aligned}
\mathcal{M}_{k}= & \left\{\left(h_{1}-h_{1}^{-1}\right) \cdots\left(h_{k}-h_{k}^{-1}\right)\left(1-a^{2}\right) a: h_{i} \in P, a \in\left(Q_{8} \times E\right) \backslash \zeta\left(Q_{8} \times E\right)\right\} \cup \\
& \left\{g\left(h_{1}-h_{1}^{-1}\right) \cdots\left(h_{k}-h_{k}^{-1}\right)\left(1-a^{2}\right) a: h_{i} \in P, a \in\left(Q_{8} \times E\right) \backslash \zeta\left(Q_{8} \times E\right)\right\} .
\end{aligned}
$$

Proof. From Theorem 2.1, we know that $N \simeq Q_{8} \times E \times P$, where $E^{2}=1, P$ is a finite $p$-group and there exists $g \in G \backslash N$ such that $G \simeq\left\langle Q_{8}, g\right\rangle \times E \times P$, $(g, x)=(g, y)=1$ and $g^{2}=x^{2}$. By Lemma 4.3 , there exists $0 \neq \widehat{P}(1-c) a \in$ $\gamma^{m(p-1)}\left((F G)^{+}\right)$for some $a \in Q_{8} \times E$. In this way, $1+m(p-1) \leq \mathrm{t}\left((F G)^{+}\right)$. Furthermore, Lemma 4.1 implies that $\mathrm{t}^{\mathrm{L}}\left((F G)^{+}\right) \leq \mathrm{t}_{\text {nil }}(P)$.

To show (ii), consider the symmetric elements $u_{i}=1-a_{i}\left(1+a_{i}^{2}\right)+x_{i}$, where $x_{i}=a_{i}\left(h_{i}+a_{i}^{2} h_{i}^{-1}\right) \in \mathcal{S}, a_{i} \in Q_{8} \times E$ and $h_{i} \in P$. Thus, $u_{i}=1+a_{i}\left(h_{i}-1\right)+a_{i}^{3}\left(h_{i}^{-1}-1\right) \in 1+F G \Delta(P)$. Since $F G \Delta(P)$ is a nilpotent ideal, we get that $1+F G \Delta(P)$ is a normal subgroup of $\mathcal{U}(F G)$ and in consequence $u_{i}$ is a unit in $F G$. We will prove, by induction, that

$$
\left(u_{1}, u_{2}, \ldots, u_{n}\right) \equiv 1+\left[x_{1}, x_{2}, \ldots, x_{n}\right] \quad\left(\bmod F G \Delta(P)^{n+1}\right) .
$$

Since $u_{1}^{-1} u_{2}^{-1} \equiv 1(\bmod F G \Delta(P))$, Lemma 4.1 implies that

$$
\begin{aligned}
\left(u_{1}, u_{2}\right) & =1+u_{1}^{-1} u_{2}^{-1}\left[u_{1}, u_{2}\right]=1+\left(u_{1}^{-1} u_{2}^{-1}-1\right)\left[u_{1}, u_{2}\right]+\left[u_{1}, u_{2}\right] \\
& \equiv 1+\left[u_{1}, u_{2}\right] \quad\left(\bmod F G \Delta(P)^{3}\right) .
\end{aligned}
$$

We recall that $\widehat{a}_{i}=1+a_{i}+a_{i}^{2}+a_{i}^{3}$ and $1+a_{i}^{2}$, for each $a_{i} \in Q_{8} \times E$, are central elements of $F G$. So

$$
\begin{aligned}
{\left[u_{1}, u_{2}\right] } & =\left[1-a_{1}\left(1+a_{1}^{2}\right)+x_{1}, 1-a_{2}\left(1+a_{2}^{2}\right)+x_{2}\right] \\
& =\left[x_{1}, x_{2}\right]+\left[a_{1}\left(1+a_{1}^{2}\right), a_{2}\left(1+a_{2}^{2}\right)\right]-\left[x_{1}, a_{2}\left(1+a_{2}^{2}\right)\right]-\left[a_{1}\left(1+a_{1}^{2}\right), x_{2}\right] \\
& =\left[x_{1}, x_{2}\right]+\left[\widehat{a}_{1}, \widehat{a}_{2}\right]-\left[x_{1}, \widehat{a}_{2}\right]-\left[\widehat{a}_{1}, x_{2}\right] \\
& =\left[x_{1}, x_{2}\right],
\end{aligned}
$$

which proves the congruence (8) when $n=2$. 
Suppose that (8), is true to $n-1$; that is

$$
\left(u_{1}, u_{2}, \ldots, u_{n-1}\right) \equiv 1+\left[x_{1}, x_{2}, \ldots, x_{n-1}\right] \quad\left(\bmod F G \Delta(P)^{n}\right) .
$$

Then, Lemma 4.1 and as $\left(u_{1}, u_{2}, \ldots, u_{n-1}\right)^{-1} u_{n}^{-1}-1 \in F G \Delta(P)$ imply

$$
\begin{aligned}
\left(u_{1}, u_{2}, \ldots, u_{n}\right) & \\
= & 1+\left(\left(u_{1}, u_{2}, \ldots, u_{n-1}\right)^{-1} u_{n}^{-1}-1\right)\left[\left(u_{1}, u_{2}, \ldots, u_{n-1}\right), u_{n}\right]+ \\
& {\left[\left(u_{1}, u_{2}, \ldots, u_{n-1}\right), u_{n}\right] } \\
\equiv & 1+\left[\left(u_{1}, u_{2}, \ldots, u_{n-1}\right), u_{n}\right] \quad\left(\bmod F G \Delta(P)^{n+1}\right) \\
\equiv & 1+\left[\left[x_{1}, x_{2}, \ldots, x_{n-1}\right], 1-a_{n}\left(1+a_{n}^{2}\right)+x_{n}\right] \quad\left(\bmod F G \Delta(P)^{n+1}\right) \\
\equiv & 1+\left[x_{1}, x_{2}, \ldots, x_{n}\right]-\left[\left[x_{1}, x_{2}, \ldots, x_{n-1}\right], \widehat{a}_{n}\right] \quad\left(\bmod F G \Delta(P)^{n+1}\right) \\
\equiv & 1+\left[x_{1}, x_{2}, \ldots, x_{n}\right] \quad\left(\bmod F G \Delta(P)^{n+1}\right),
\end{aligned}
$$

and the statement (8) is true for all $n \geq 2$.

Let $n=\mathrm{t}_{\text {nil }}(P)-1$. If $\mathrm{t}\left((F G)^{+}\right)=\mathrm{t}_{\text {nil }}(P)$, then there are $x_{1}, \ldots, x_{n} \in \mathcal{S}$ such that $\left[x_{1}, \ldots, x_{n}\right] \neq 0$. Thus, by the congruence $(8), \gamma_{n}\left(\mathcal{U}^{+}(F G)\right) \neq 1$. So $n \leq \operatorname{cl}\left(\mathcal{U}^{+}(F G)\right)$. Moreover, we know that $\operatorname{cl}\left(\mathcal{U}^{+}(F G)\right)<\mathrm{t}^{\mathrm{L}}\left((F G)^{+}\right) \leq$ $\mathrm{t}_{\mathrm{nil}}(P)=n+1$ and we get (ii).

Assume that $P$ is powerful. Then, by Lemma 4.4, we obtain

$$
x_{n, n} f_{1, \ldots, n}(1-c) a \equiv 2^{n} f_{1, \ldots, n}(1-c) a \quad\left(\bmod F G \Delta(P)^{n+1}\right) .
$$

Furthermore, if $h_{i}-1 \in \Delta(P)^{k_{i}}$, then by (2)

$$
h_{i}-h_{i}^{-1}=\left(h_{i}-1\right)-\left(h_{i}^{-1}-1\right) \equiv 2\left(h_{i}-1\right) \quad\left(\bmod \Delta(P)^{k_{i}+1}\right),
$$

thus

$$
\begin{aligned}
x_{n, n} f_{1, \ldots, n}(1-c) a & \equiv 2^{n}\left(h_{1}-h_{1}^{-1}\right) \cdots\left(h_{n}-h_{n}^{-1}\right)(1-c) a \\
& \equiv 2^{2 n}\left(h_{1}-1\right) \cdots\left(h_{n}-1\right)(1-c) a \quad\left(\bmod F G \Delta(P)^{n+1}\right) .
\end{aligned}
$$

It is clear that, if $n<\mathrm{t}_{\text {nil }}(P)$, there exist $h_{1}, \ldots, h_{n} \in P$ such that $\prod_{i=1}^{n}\left(h_{i}-\right.$ $1) \neq 0$, and then $x_{n, n} M_{n} \neq 0$. Thus, $\mathrm{t}_{\text {nil }}(P) \leq \mathrm{t}\left((F G)^{+}\right)$and (iii) follows.

Finally, assume that $P$ is abelian. Let $a, b \in\left(Q_{8} \times E\right) \backslash \zeta\left(Q_{8} \times E\right)$, and $h_{1}, h_{2} \in P$, such that $(a, b) \neq 1$. Then

$$
\begin{aligned}
{\left[a\left(h_{1}+a^{2} h_{1}^{-1}\right), b\left(h_{2}+b^{2} h_{2}^{-1}\right)\right] } & =\left(h_{2}+b^{2} h_{2}^{-1}\right)\left(h_{1}+a^{2} h_{1}^{-1}\right)[a, b] \\
& =\left(h_{2}-h_{2}^{-1}\right)\left(h_{1}-h_{1}^{-1}\right)(1-c) a b .
\end{aligned}
$$

If $\alpha \in F P, h \in P$, then

$$
\begin{aligned}
{\left[\alpha(1-c) a, b\left(h+b^{2} h^{-1}\right)\right] } & =\alpha(1-c)\left(h+b^{2} h^{-1}\right)[a, b] \\
& =\alpha\left(h-h^{-1}\right)(1-c)^{2} a b=2 \alpha\left(h-h^{-1}\right)(1-c) a b .
\end{aligned}
$$




$$
\begin{aligned}
{\left[a\left(h_{1}+a^{2} h_{1}^{-1}\right), b g\left(h_{2}-h_{2}^{-1}\right)\right] } & =\left(h_{2}-h_{2}^{-1}\right)\left(h_{1}+a^{2} h_{1}^{-1}\right)[a, b g] \\
& =g\left(h_{2}-h_{2}^{-1}\right)\left(h_{1}+c h_{1}^{-1}\right)(1-c) a b \\
& =g\left(h_{2}-h_{2}^{-1}\right)\left(h_{1}-h_{1}^{-1}\right)(1-c) a b,
\end{aligned}
$$

and

$$
\begin{aligned}
{\left[a g\left(h_{1}-h_{1}^{-1}\right), b g\left(h_{2}-h_{2}^{-1}\right)\right] } & =\left(h_{2}-h_{2}^{-1}\right)\left(h_{1}-h_{1}^{-1}\right)[a g, b g] \\
& =\left(h_{2}-h_{2}^{-1}\right)\left(h_{1}-h_{1}^{-1}\right) g^{2}(1-c) a b \\
& =-\left(h_{2}-h_{2}^{-1}\right)\left(h_{1}-h_{1}^{-1}\right)(1-c) a b .
\end{aligned}
$$

The equations (11), (12) and (13) imply that $\gamma^{2}\left((F G)^{+}\right)=\mathcal{M}_{2}$. Suppose that

$\gamma^{n-1}\left((F G)^{+}\right)=\mathcal{M}_{n-1}$ for some $n \geq 3$. Take $\alpha \in F P, h \in P$ and $a, b \in\left(Q_{8} \times E\right) \backslash \zeta\left(Q_{8} \times E\right)$, such that $(a, b) \neq 1$. We get the following equalities:

$$
\begin{aligned}
{\left[\alpha(1-c) a, g b\left(h-h^{-1}\right)\right] } & =\alpha\left(h-h^{-1}\right)(1-c)[a, g b] \\
& =g \alpha\left(h-h^{-1}\right)(1-c)[a, b] \\
& =g \alpha\left(h-h^{-1}\right)(1-c)^{2} a b \\
& =2 g \alpha\left(h-h^{-1}\right)(1-c) a b
\end{aligned}
$$

and

$$
\begin{aligned}
{\left[g \alpha(1-c) a, g b\left(h-h^{-1}\right)\right] } & =g^{2} \alpha\left(h-h^{-1}\right)(1-c)[a, b] \\
& =c \alpha\left(h-h^{-1}\right)(1-c)[a, b] \\
& =c \alpha\left(h-h^{-1}\right)(1-c)^{2} a b \\
& =-2 \alpha\left(h-h^{-1}\right)(1-c) a b .
\end{aligned}
$$

By substituting $f_{1, \ldots, n-1}$ for $\alpha$ in (11), (14) and (15), we get that $\left[\mathcal{M}_{n-1},(F G)^{+}\right]=\mathcal{M}_{n}$ and therefore

$$
\gamma^{n}\left((F G)^{+}\right)=\left[\gamma^{n-1}\left((F G)^{+}\right),(F G)^{+}\right]=\left[\mathcal{M}_{n-1},(F G)^{+}\right]=\mathcal{M}_{n},
$$

as we wanted to prove.

\section{Acknowledgements}

The results of this paper are part of the author's Ph.D. thesis, at the Instituto de Matemática e Estatística of the Universidade de São Paulo, under the guidance of Prof. César Polcino Milies. This work was partially supported by CAPES and CNPq. proc. 141857/2011-0 of Brazil. 


\section{References}

1. Z. Balogh and T. Juhász, Derived lengths of symmetric and skew symmetric elements in group algebras, JP J. Algebra Number Theory Appl. 12 (2008), no. 2, 191-203. MR 2500081 (2010b:16045)

2. _ Nilpotency class of symmetric units of group algebras, Publ. Math. Debrecen 79 (2011), no. 1-2, 171-180. MR 2850041 (2012h:16048)

3 . Nilpotency indices of symmetric elements of group algebras, Comm. Algebra 40 (2012), no. 11, 4283-4294.

4. V. Bovdi and E. Spinelli, Modular group algebras with maximal Lie nilpotency indices, Publ. Math. Debrecen 65 (2004), no. 1-2, 243-252. MR 2075267 (2005d:16040)

5. John H. Castillo and C. Polcino Milies, Lie properties of symmetric elements under oriented involutions, Comm. Algebra 40 (2012), no. 12, 4404-4419. MR 2989653

6. A. Giambruno and S. K. Sehgal, Lie nilpotence of group rings, Comm. Algebra 21 (1993), no. 11, 4253-4261. MR 1238157 (94g:20008)

7. S. A. Jennings, The structure of the group ring of a p-group over a modular field, Trans. Amer. Math. Soc. 50 (1941), 175-185. MR 0004626 (3,34f)

8. G. T. Lee, Group rings whose symmetric elements are Lie nilpotent, Proc. Amer. Math. Soc. 127 (1999), no. 11, 3153-3159. MR 1641124 (2000b:16052)

9. $\quad$ The Lie $n$-Engel property in group rings, Comm. Algebra 28 (2000), no. 2, 867-881. MR 1736769 (2001b:16027)

10. Group identities on units and symmetric units of group rings, Algebra and Applications, vol. 12, Springer-Verlag London Ltd., London, 2010. MR 2723223

11. S. P. Novikov, Algebraic construction and properties of Hermitian analogs of $K$ theory over rings with involution from the viewpoint of Hamiltonian formalism. Applications to differential topology and the theory of characteristic classes. I. II, Izv. Akad. Nauk SSSR Ser. Mat. 34 (1970), 253-288; ibid. 34 (1970), 475-500. MR 0292913 (45 \#1994)

12. I. B. S. Passi, D. S. Passman, and S. K. Sehgal, Lie solvable group rings, Canad. J. Math. 25 (1973), 748-757. MR 0325746 (48 \#4092)

13. C. Polcino Milies and S. K. Sehgal, An introduction to group rings, Algebras and Applications, vol. 1, Kluwer Academic Publishers, Dordrecht, 2002. MR 1896125 (2003b:16026)

14. S. K. Sehgal, Topics in group rings, Monographs and Textbooks in Pure and Applied Math., vol. 50, Marcel Dekker Inc., New York, 1978. MR 508515 (80j:16001)

15. R. K. Sharma and V. Bist, A note on Lie nilpotent group rings, Bull. Austral. Math. Soc. 45 (1992), no. 3, 503-506. MR 1165157 (93g:20011) 